Supporting Information

\title{
Decisive Influence of Hydrophobic Side Chains of Polyesters on Thermo-induced Gelation of Triblock Copolymer Aqueous Solutions
}

Kaiting Wu, Xiaobin Chen, Siyi Gu, Shuquan Cui, Xiaowei Yang, Lin Yu*, Jiandong Ding

State Key Laboratory of Molecular Engineering of Polymers, Department of Macromolecular Science, Fudan University, Shanghai 200438, China 


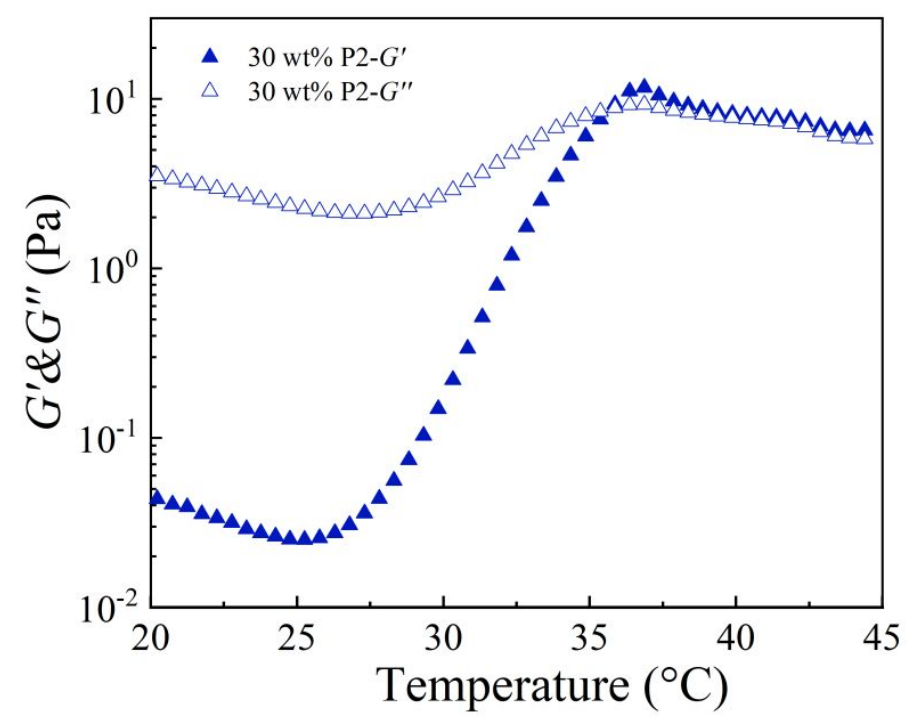

Figure S1. Elastic moduli $\left(G^{\prime}\right)$ and viscous moduli $\left(G^{\prime \prime}\right)$ of the aqueous solution of P2 triblock copolymer as a function of temperature. The polymer concentration was set as $30 \mathrm{wt} \%$.

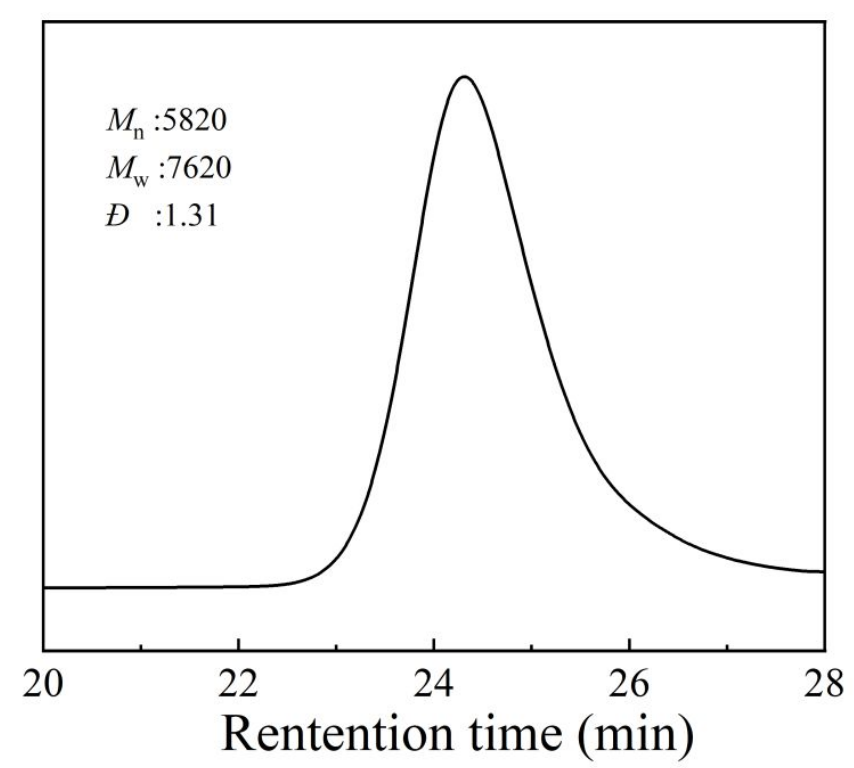

Figure S2. GPC trace of RB-PCOL-PEG-PCOL-RB. 


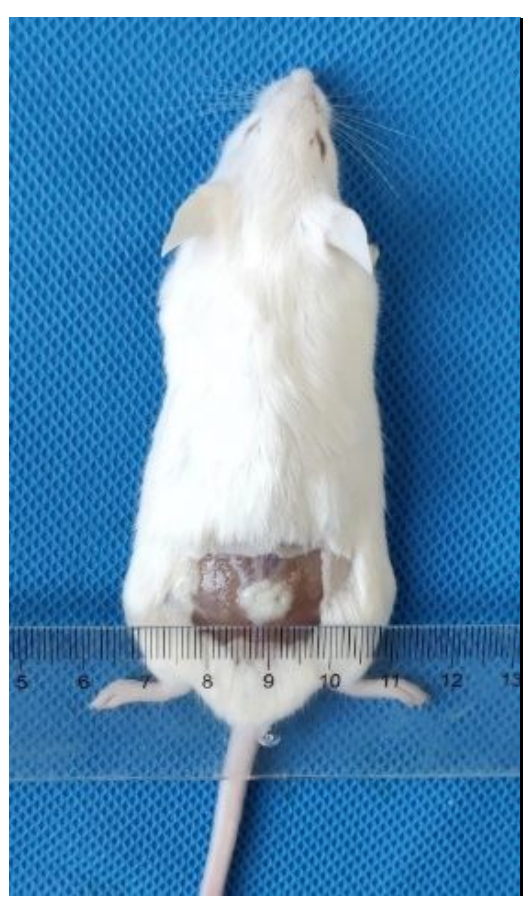

Figure S3. Optical image of the PCOL-PEG-PCOL (P3) hydrogel (30 wt\%) 3 weeks after subcutaneous injection into a mouse. 